\title{
The Chaperone Activity of Clusterin is Dependent on Glycosylation and Redox Environment
}

\author{
Philipp Rohne ${ }^{a}$ Hans Prochnow ${ }^{a}$ Steven Wolfa Benjamin Renner ${ }^{a}$ \\ Claudia Koch-Brandt ${ }^{\mathrm{a}}$ \\ anstitute of Pharmacy and Biochemistry, Therapeutical Life Sciences, Johannes Gutenberg -University \\ Mainz, Mainz, Germany
}

\section{Key Words}

Chaperones • Clusterin • Apolipoprotein J - Glycosylation • Furin-like proprotein convertases - Redox biology $\cdot$ Retro-translocation $•$ Proteostasis network $•$ Heat shock

\begin{abstract}
Background/Aims: Clusterin (CLU), also known as Apolipoprotein J (ApoJ) is a highly glycosylated extracellular chaperone. In humans it is expressed from a broad spectrum of tissues and related to a plethora of physiological and pathophysiological processes, such as Alzheimer's disease, atherosclerosis and cancer. In its dominant form it is expressed as a secretory protein (secreted CLU, sCLU). During its maturation, the sCLU-precursor is $\mathrm{N}$-glycosylated and cleaved into an $\alpha$ - and a $\beta$-chain, which are connected by five symmetrical disulfide bonds. Recently, it has been demonstrated that besides the predominant $\mathrm{SCLU}$, rare intracellular CLU forms are expressed in stressed cells. Since these forms do not enter or complete the secretory pathway, they are not proteolytically modified and show either no or only core-glycosylation. Due to their sparsity, these intracellular forms are functionally poorly characterized. To evaluate the function(s) of these stress-related intracellular forms, we investigate for the first time the impact of proteolytic cleavage, differential glycosylation and the influence of the redox environment on the chaperone activity of CLU. Methods: Non-cleavable sCLU was generated by expression from a mutant construct of $\mathrm{sCLU}$, in which the furin-like proprotein convertase (PC) recognition site was modified. After purification of recombinant uncleaved SCLU from the medium of over-expressing cells, we performed chaperone activity assays to compare the activities of wild-type (cleaved) and uncleaved mutant sCLU. Additionally, this approach enabled us to investigate the role of carbohydrates, the proteolytic maturation and reducing conditions on CLU chaperone activity. Further, we characterized the differentially treated CLU forms by using MALDI-TOF, CD-spectroscopy and Western blotting in addition to the functional assay. Results: We show that the PC-cleavage is dispensable for SCLU chaperone activity. Moreover, our data demonstrate that while fully deglycosylated SCLU lacks chaperone activity, partially deglycosylated sCLU is still capable of solubilizing target proteins. Most importantly, we here demonstrate for the first time that uncleaved SCLU is highly sensitive


towards reducing conditions. Conclusions: Our study provides evidence that unglycosylated intracellular CLU forms cannot exhibit a chaperone activity compared to sCLU. Additionally, we support recent postulates that glycosylated intracellular CLU forms may act as a redox sensor under oxidative stress conditions. Furthermore, we conclude that the proteolytic cleavage of sCLU is important to maintain full chaperone activity, i.e. in the presence of necrosis.

Copyright (c) 2014 S. Karger AG, Basel

\section{Introduction}

Clusterin (CLU), also known as Apolipoprotein J, is a ubiquitously expressed protein found in nearly all fluids and tissues of the human body. It is one of only few known extracellular chaperones acting outside the living cell $[1,2]$.

Secretory Clusterin (sCLU) is synthesized as a pre-pro-protein consisting of 449 amino acids. The N-terminal 22 amino acids represent a hydrophobic signal sequence for segregation into the endoplasmic reticulum (ER), where the nascent polypeptide is processed to a $60 \mathrm{kDa}$ high mannose single chain precursor (pre-secretory CLU, psCLU) possessing five intramolecular disulfide bonds [3-5]. After translocation to the Golgi-apparatus, psCLU is terminally glycosylated with the mass percentage rate of carbohydrates reaching up to $30 \%$, depending on the tissue [6]. Upon transport to the cell surface, the precursor is cleaved by a furin-like proprotein convertase (PC) producing an $\mathrm{N}$-terminal $\alpha$-chain and a C-terminal $\beta$-chain [7]. Mature secretory CLU (sCLU) is secreted as a heterodimeric protein with an apparent molecular weight (MW) of 70-80 kDa [8, 9].

Despite being a water-soluble protein, sCLU has a prominent hydrophobic character. Under physiological conditions the protein is usually present in higher oligomeric forms rather than as monomers, dimers or tetramers [10-13]. In silico analyses suggest that sCLU contains five amphipathic $\alpha$-helices which contribute more than one third to the protein's secondary structure as determined by CD- and IR-spectroscopy. Yet another third of the secondary structure consists of unordered regions [13-17]. It is assumed that sCLU contains a significant amount of so-called molten globule-like domains. These are sections which might have a defined secondary structure but lack any ordered tertiary structure. Hence, within molten globule-like domains, hydrophobic amino acids can be exposed on the surface of the protein. This property is crucial for a key role regarding the function of sCLU and is also found in other intrinsically disordered proteins such as Calcineurin, Tau-protein or p53 $[2,18,19]$.

Like other chaperones, CLU-mRNA is upregulated as part of the so-called heat shock response owing to a heat shock element in its promotor region [20-22]. The heat shock response is a cellular defense program triggering the upregulation of a broad spectrum of distinct proteins such as proteolytic enzymes, detoxifying proteins or molecular chaperones [23]. These protect the cell against the devastating effects of UV-light, ionizing radiation or oxidizing reagents, which on their part cause an increase in reactive oxygen species (ROS) and further lead to an accumulation of misfolded proteins [24-26]. Such interference in protein homeostasis may result in proteotoxic stress. Prolonged proteotoxic stress is regarded as a hallmark of a variety of diseases including amyloidosis, ischemia or cancer and as a promoter of cellular aging in general. Interestingly, CLU was found to be related to all of these pathological processes [27-32]. As a molecular chaperone sCLU is able to counteract proteotoxic stress by binding to intermediate folding states of proteins that are in the process of denaturing. Thus, it prevents their uncontrolled aggregation and keeps the accrued complexes in solution in an ATP-independent manner [33, 34]. This is a feature sCLU shares with small heat shock proteins (sHSPs) like Hsp27, which are found inside the cell [25]. The binding to the misfolded proteins occurs via hydrophobic interactions between the molten globule-like domains of sCLU and the exposed hydrophobic regions of the target proteins [2, 35-37]. Furthermore, sCLU not only has the ability to solubilize misfolded proteins, but also to promote their removal from the extracellular space. This, in contrast, is believed to be mediated by a few organized regions within the sCLU-protein which can bind 
to certain receptors of the LRP (lipoprotein receptor-related protein)-receptor family on the surface of vital cells $[9,17,38-40]$. Thereby, sCLU initiates the endocytic uptake and removal of misfolded protein aggregates, thus rendering it a key player during the homeostasis of the proteome, a process which is also termed proteostasis [24, 41-43]. Furthermore, recent studies suggest that sCLU is an important player for modulating MAPK/Erk and PI3K/Akt signaling pathways. However, the effects differ depending on cell type [44-48].

Besides extracellular sCLU, non-secreted forms of Clusterin are present in minor amounts in stressed cells. It was recently shown that these (for the most part) represent unglycosylated cytosolic proteins which are not proteolytically processed into $\alpha$ - and $\beta$-chains. They either represent the sCLU pre-pro-protein that fails to be segregated into the ER of stressed cells, alternative spliced CLU or CLU originated from an internal translation initiation at different start sites on the CLU-mRNA downstream of the ER-leader. The latter two events result in protein isoforms lacking the signal sequence for segregation into the ER [22]. Yet another non-secreted form of CLU is believed to emerge from retro-translocation of immaturely glycosylated psCLU from the ER-Golgi network to the cytosol. This event was observed in cells being exposed to ER-stress conditions induced by thapsigargin, MG-132 or paclitaxel. For the retro-translocation of CLU, the ER-resident co-chaperone GRP78 (Bip) seems to play a pivotal role $[7,49]$.

Up to now it is unclear whether non-secreted forms of CLU also exhibit a chaperone activity causing them to become an intracellular pendant to extracellular sCLU. This hypothesis is strengthened by observations, which imply that the glycosylation state of CLU does not seem to have any effect on its chaperone activity [16]. Furthermore, it is currently unknown whether the proteolytic maturation is a prerequisite of CLU function [50]. This must be taken into consideration before assigning non-secreted CLU forms a chaperone activity.

In this work we investigate whether intracellular CLU forms present in stressed cells can exert chaperone activity. This information will be useful to evaluate a potential role of intracellular CLU forms in a large variety of diseases.

\section{Material and Methods}

Cloning, mutation and transfection of clusterin expression plasmids Human clusterin (CLU) full length cDNA variant 1 [51] was cloned into pcDNA6-V5/6xHis (Life Sciences). The vector system adds aV5-epitope and a His-tag to the C-terminal end of the recombinant proteins. To mutate the putative recognition site for PC (amino acid-motif: RIVR), the following primers were used for site-directed in vitro mutagenesis: 5'-CCGCATCGTCCAGAGCTTGATGC-3' (forward) and 5'-GCATCAAGCTCTGGACGATGCGG-3' (reverse) to realize a RIVR to RIVQ substitution or 5'-GTCCCGCATCACCCGCAGCTTG-3' (forward) and 5'-CAAGCTGCGGGTGATGCGGGAC-3' (reverse) to allow for replacement of RIVR to RITR. As expression host, HEK-293 cells were chosen. After growth at $37^{\circ} \mathrm{C}$ in a humidified atmosphere with $5 \% \mathrm{CO}_{2}$ and medium containing 10\% FBS, the cells were transfected. The insertion of $2 \mu \mathrm{g}$ plasmid DNA into the host cells was carried out using OptiMEM ${ }^{\circledR}$ (Life Technologies) and Turbofect ${ }^{\mathrm{TM}}$ in vitro transfection reagent (Thermo Scientific) in accordance with the manufacturer's protocol. To examine the transfection success, cells were cultivated for an additional $24 \mathrm{~h}$ under serum-free conditions. Supernatants and lysates were taken and sCLU expression was examined by Western blotting. Stable cell clones were obtained by selection in presence of $10 \mu \mathrm{g} / \mathrm{mL}$ Blasticidin S hydrochloride (Carl Roth).

\section{Preparation of cell lysates and supernatants}

After serum-free cultivation of the transfected cells for $24 \mathrm{~h}$, cell culture supernatants were collected and floating cells were removed by centrifugation for $10 \mathrm{~min}$ at $1000 \mathrm{xg}$. Cells were gathered in ice-cold lysis buffer (50 mM Tris/HCl [pH 8], $150 \mathrm{mM} \mathrm{NaCl,} \mathrm{1 \%} \mathrm{Triton}{ }^{\circledR} \mathrm{X}-100$ ) containing a protease inhibitor (Complete mini, Roche) and incubated at $4^{\circ} \mathrm{C}$ under gentle agitation for $30 \mathrm{~min}$. Subsequently, the lysates were centrifuged for another $30 \mathrm{~min}\left(20.000 \mathrm{xg}, 4^{\circ} \mathrm{C}\right)$ to remove insoluble material. Cleared cell-lysates were transferred to fresh tubes and protein concentration was quantified by using a Bradford assay. 


\section{SDS-PAGE, Western blot analyses and Coomassie staining}

SDS-PAGEs with Acryl-/Bisacrylamide concentrations of 9, 10 or $12.5 \%(\mathrm{v} / \mathrm{v})$ were performed (see Figures). Samples were diluted in water and $5 \mathrm{x}$ loading buffer $(0.225 \mathrm{M}$ Tris [pH 6.8], 50\% [v/v] glycerol, $5 \%[\mathrm{w} / \mathrm{v}] \mathrm{SDS}, 0.05 \%$ bromphenol blue) with or without $0.25 \mathrm{M}$ DTT. For Western blotting $50 \mu \mathrm{g}$ cell lysates or $200 \mathrm{ng}$ purified/treated sCLU per lane was used. For immuno detection, SDS-PAGEs were blotted onto nitrocellulose membranes using a Mini Trans-Blot ${ }^{\circledR}$ Electrophoretic Transfer Cell (Bio-Rad). Membranes were blocked with $5 \%$ milk powder/PBS solution containing $0.1 \%(\mathrm{v} / \mathrm{v})$ Tween ${ }^{\circledR} 20$. We used the anti-V5 antibody (Life Technologies) for detection of the recombinant CLU $\beta$-chain and the clone 41D antibody (Millipore) to detect the sCLU $\alpha$-chain. Hereafter, respective bands were visualized by incubation with HRPlinked secondary antibodies which allow for chemiluminescence detection. All antibodies were diluted in $5 \%$ milk powder/PBS solution containing $0.1 \%(\mathrm{v} / \mathrm{v})$ Tween ${ }^{\circledR} 20$ and incubated on the membranes overnight at $4^{\circ} \mathrm{C}$. As a loading control $0.5 \%(\mathrm{w} / \mathrm{v})$ Ponceau S diluted in $1 \%(\mathrm{v} / \mathrm{v})$ glacial acetic acid was used. To determine the purity of recombinant sCLU, $4 \mu \mathrm{g}$ sCLU were loaded onto SDS-PAGEs and stained with Coomassie Brilliant Blue overnight (CBB-R250 (Roth) diluted in 20\% methanol). Gels were destained in a $20 \%$ methanol solution.

\section{Metal ion affinity chromatography}

HEK-293 cells overexpressing either cleaved or uncleaved sCLU were cultivated to a confluency of $100 \%$ and subsequently serum-starved for at least $60 \mathrm{~h}$. Dead or detached cells were removed from the supernatant by centrifugation $\left(1.000 \mathrm{xg}, 30 \mathrm{~min}, 4^{\circ} \mathrm{C}\right)$. The DMEM-based buffer of the cleared sCLU-enriched supernatant was substituted by LEW-buffer ( $50 \mathrm{mM} \mathrm{Na}{ }_{2} \mathrm{HPO}_{4} / \mathrm{NaH}_{2} \mathrm{PO}_{4}$ [pH 8], $300 \mathrm{mM} \mathrm{NaCl}$ ) containing $7 \%$ glycerol via a Minimate ${ }^{\mathrm{TM}}$ TFF Capsule 30 or $50 \mathrm{~K}$ (PALL). The obtained solution was subjected to a FPLC-column (GE Healthcare) packed with Protino ${ }^{\circledR} \mathrm{Ni}$-IDA resin (Machery-Nagel). After two washing steps with LEW-buffer (the second step with additional $25 \mathrm{mM}$ Imidazol), the bound sCLU fractions were eluted by using LEW-buffer containing $250 \mathrm{mM}$ Imidazole. Imidazole was removed by substituting the LEWbuffer against PBS with an Amicon Ultra-15 Centrifugal Filter Unit, 50 NMWL (Millipore). The final protein concentration was adjusted to $1 \mu \mathrm{g} / \mu \mathrm{L}$ as determined by a Bradford assay.

\section{Protein deglycosylation}

Partial deglycosylation was achieved by treatment of sCLU together with three exoglycosidases. $100 \mu \mathrm{g}$ sCLU diluted in $100 \mu \mathrm{L}$ PBS was incubated with $4.2 \mathrm{U} / \mu \mathrm{L} \alpha 2,3$-neuraminidase, $0.7 \mathrm{U} / \mu \mathrm{L} \beta 1$, 4 -galactosidase and $0.3 \mathrm{U} / \mu \mathrm{L} \beta$ - $N$-acetylglucosaminidase at $37^{\circ} \mathrm{C}$ for $12 \mathrm{~h}$ under gentle agitation at $\mathrm{pH} 6$. Subsequently, the buffer was substituted against PBS with a Vivaspin 4 5,000 MWCO PES (Sartorius). For full deglycosylation, sCLU $(25-400 \mu \mathrm{g})$ was treated with various concentrations of PNGase F (see Figures) under the same conditions. All glycosidases were purchased from NEB.

\section{MALDI-TOF analysis}

To determine the success of full sCLU deglycosylation, MALDI-TOF analyses were carried out by dilution in a sialic acid matrix. The analyses occurred using a Shimadzu CFR Axima (Kratos Analytica).

\section{Chaperone activity assay}

The examination of CLU chaperone activity was carried out as shown previously [37]. Soluble cell extract was obtained from HEK-293 cells by freeze and thaw of a suspension of $2 \times 10^{6}$ cells $/ \mathrm{mL}$ in $10 \mathrm{mM}$ $\mathrm{NaH}_{2} \mathrm{PO}_{4}$ ( $\mathrm{pH} 7$ ) with subsequent centrifugation $(20.000 \mathrm{xg}, 1 \mathrm{~h})$ to remove insoluble material. Protein concentration was determined by the Bradford assay. Catalase $(1 \mathrm{mg} / \mathrm{mL}$; Sigma Aldrich) or soluble cell extract $(750 \mu \mathrm{g} / \mathrm{mL})$ was mixed with CLU $(75 \mu \mathrm{g} / \mathrm{mL}$ or $100 \mu \mathrm{g} / \mathrm{mL}$ for the experimentin Fig. 6B, respectively) or BSA ( $83 \mu \mathrm{g} / \mathrm{mL})$ as a non-chaperone control in a total volume of $100 \mu \mathrm{L}$ reaction buffer $\left(50 \mathrm{mM} \mathrm{NaH}_{2} \mathrm{PO}_{4}\right.$ with or without $5 \mathrm{mM}$ DTT). The assay was performed at $50^{\circ} \mathrm{C}$ for 60 or $150 \mathrm{~min}$, respectively, while protein precipitation was concomitantly monitored by detecting the optical density at $360 \mathrm{~nm}$ (turbidity) in an ELISA reader PowerWave XS (Biotek). All data were corrected by the absorption of plain assay buffer.

\section{Circular Dichroism}

To determine the relative percentage of sCLU secondary structures, CD analyses were performed. The measurements were carried out in a Jasco J-815 spectropolarimeter with $0.1 \mu \mathrm{g} / \mu \mathrm{L}$ protein diluted in $10 \mathrm{mM} \mathrm{NaH}_{2} \mathrm{PO}_{4}(\mathrm{pH} 7)$ in a step-scan mode using $1.0 \mathrm{~mm}$ path length quartz cells (Hellma-analytics) 
at $20^{\circ} \mathrm{C}$. CD-Spectra were obtained via a step resolution of $1.0 \mathrm{~nm}$ and a bandwidth of $5 \mathrm{~nm}$ at a sensitivity of 100 mdeg. The correction of an average spectrum acquired from three scans was performed in relation to the blank buffer and smoothed with a Savitzky-Golai convolution using Spectra Manager (Jasco). For secondary structure prediction, the CDSSTR algorithm was used (Whitmore and Wallace 2007). Molecular masses of $70 \mathrm{kDa}$ for fully glycosylated, $61.4 \mathrm{kDa}$ for partially and $55.6 \mathrm{kDa}$ for fully deglycosylated sCLU were assumed. All sCLU forms are considered to have a length of 478 amino acids (427 aa+51 aa tag).

\section{Results}

Proteolytic cleavage of CLU is not necessary for its chaperone activity

Since intracellular forms of CLU do not undergo proteolytic cleavage into a- and ßchains, we first set out to investigate the impact of the proteolytic cleavage on the chaperone activity. We therefore generated recombinant cDNA constructs encoding either wild-type (cleaved) sCLU or sCLU having a modified protease-recognition site. In the latter case we converted the amino acid sequence of the putative recognition site from RIVR to RIVQ and RITR, respectively (Fig. 1A). Following transfection into HEK-293 cells and Western blot analyses, we observed that the CLU-expression pattern generated from the cells hosting the "RITR"-construct showed no difference compared to cells expressing wild-type sCLU. This mentioned CLU expression pattern of cell lysates shows a prominent band at approximately $70 \mathrm{kDa}$ corresponding to the uncleaved CLU precursor (psCLU) in the ER-Golgi-network.

Fig. 1. Cloning, expression and characterization of recombinant cleaved and uncleaved SCLU. A. Graphical depiction of the amino acid sequence of recombinant CLU: highlighted are $\alpha$ - and $\beta$-chain, the furinlike proprotein convertase (PC) recognition site, the recombinant penta-His tag and the V5-epitope. The PC recognition site was modified by site-directed in vitro mutagenesis as described in Material \& Methods. B. West-

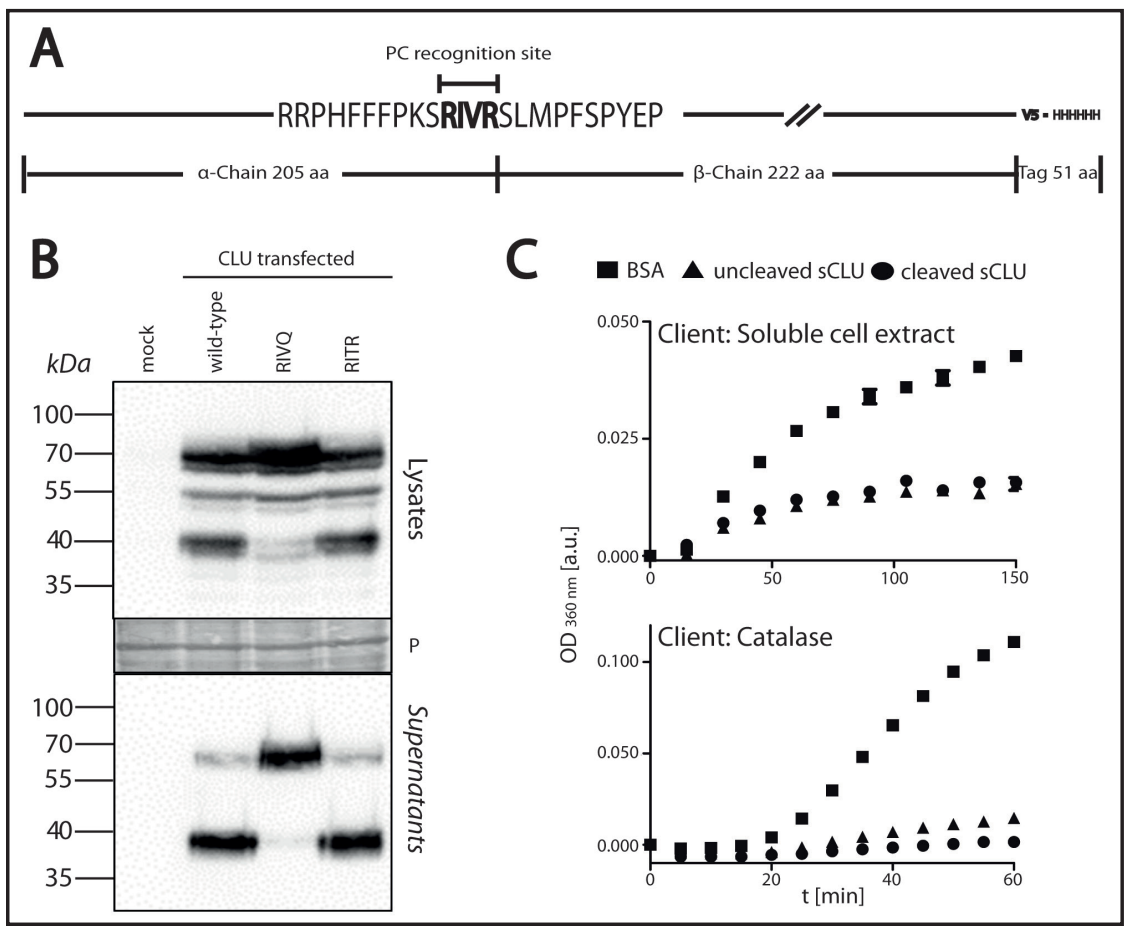

ern blot analysis (10\% SDS-PAGE under reducing conditions) of lysates and cell-culture supernatants of HEK-293 cells transiently transfected with recombinant wild-type and mutant CLU cDNA-constructs cloned into pcDNA6. As a mock control, blank pcDNA6-transfected cells were analyzed. The detection of CLU was realized by using the anti-V5 antibody corresponding to the recombinant tag. Ponceau-Staining (P) was used as a loading control. C. Chaperone activity assays: purified sCLU proteins were incubated with cleared soluble cell extract for $150 \mathrm{~min}$ (upper panel) or with Catalase for $60 \mathrm{~min}$ (lower panel) under non-reducing conditions. As control, the clients were incubated with BSA under the same conditions. Data are plotted in intervals of 5 min (Catalase) or 15 min (soluble cell extract). Data points are means of three measurements, error bars correspond to the standard deviation. The data shown are representative results of at least three independent experiments. 
Another striking band is the $\beta$-chain of the mature cleaved secretory CLU (sCLU) at around $40 \mathrm{kDa}$. In addition, an underrepresented band at $55 \mathrm{kDa}$ is also visible. This protein corresponds to the unglycosylated, uncleaved CLU forms in the cytosol of stressed cells as described by Prochnow et al. [22]. In contrast, the supernatant of cells transfected with wildtype CLU or "RITR"-constructs revealed that the majority of CLU secreted from the cell is represented by the $\beta$-chain at $40 \mathrm{kDa}$. No $55 \mathrm{kDa}$ intracellular CLU were detected and only a minor amount of uncleaved psCLU is co-secreted from the cell, probably caused due to the overexpression.

In lysates and supernatants of cells transfected with the construct carrying the RIVR to RIVQ mutation, no band at $40 \mathrm{kDa}$ corresponding to the cleaved mature sCLU is detectable. As expected, the $55 \mathrm{kDa}$ band of the cytosolic CLU form(s) was again visible. Interestingly, the sCLU form generated from the RIVR to RVQ substitution (hereafter referred to as uncleaved sCLU) is still being secreted and detectable in the cell culture supernatant analogous to cleaved CLU (Fig. 1B).

In a next step, both cleaved and uncleaved recombinant sCLU were purified via metal ion chromatography and processed to assay chaperone activity (see Material and Methods). The chaperone activity assays were performed at $50^{\circ} \mathrm{C}$ under non-reducing conditions using soluble cell extract as a complex ligand and Catalase as a defined client protein. During incubation at $50^{\circ} \mathrm{C}$, the client proteins start to denature forming a precipitate which results in an increased turbidity of the solution (monitored at $\mathrm{OD}_{360 \mathrm{~nm}}$ ). Interestingly, uncleaved sCLU showed no significant difference in activity compared to cleaved sCLU: both forms solubilized client proteins in the same manner. As expected, the precipitation of the client proteins was not inhibited by BSA, which served as a control.

Core-glycosylated forms of cleaved and uncleaved SCLU display chaperone activity

Having shown that the proteolytic processing is dispensable for chaperone activity of sCLU, we next set out to characterize the role of $\mathrm{N}$-linked carbohydrates in this process. Cleaved and uncleaved sCLU were treated with the exoglycosidases $\alpha 2,3$-neuraminidase,

Fig. 2. Partial deglycosylation and characterisation of cleaved and uncleaved sCLU. A. Graphical depiction of the polysaccharide branches: shown are constituent carbohydrates and the cleavage sites of the exoglycosidases used. The treatment was carried out by incubating sCLU together with the exoglycosidases $\alpha 2,3$-neuraminidase, $\beta 1,4$-galactosidase and $\beta$-N-acetylglucosaminidase as described in Material and Methods. B. Western blot analysis (9\% SDS-PAGE under non-reducing condi-

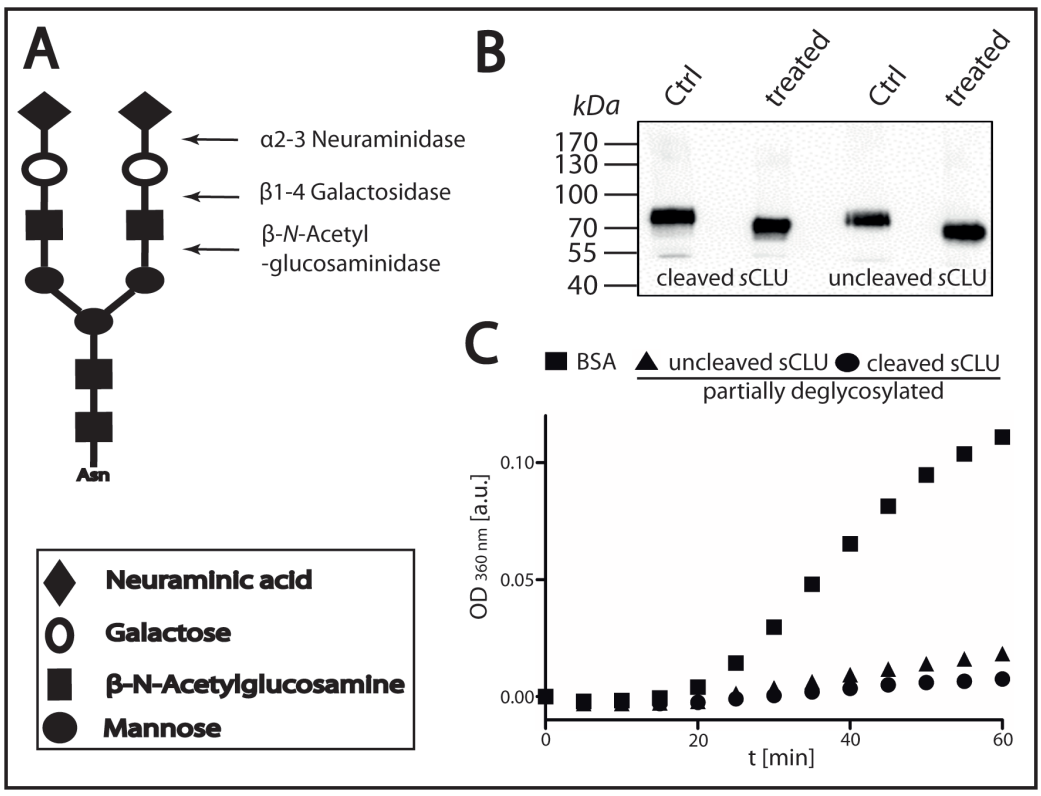
tions) of cleaved and uncleaved sCLU incubated with or without exoglycosidases (treated). The detection of CLU was realized by using the anti-V5 antibody corresponding to the recombinant tag. C. Chaperone activity assays: purified treated sCLU fractions were incubated with Catalase for 60 min under non-reducing conditions. As a control, Catalase was incubated with BSA under the same conditions. Data are plotted in intervals of $5 \mathrm{~min}$. Data points are means of two to three measurements, error bars correspond to the standard deviation. The data shown are representative results of at least two to three independent experiments. 
$\beta 1,4$-galactosidase and $\beta$ - $N$-acetylglucosaminidase resulting in sCLU retaining only the core carbohydrates (Fig. 2A). As shown in Fig. 2B, this treatment resulted in a shift in the apparent molecular weight of approximately $10 \mathrm{kDa}$ for both cleaved and uncleaved sCLU on a non-reducing SDS-PAGE. However, when performing chaperone activity assays of the core-glycosylated sCLU proteins, an activity similar to that of the fully glycosylated proteins was observed. Hence, irrespective of the proteolytic maturation state of sCLU, the terminal sugars are dispensable for its activity. (Fig. 2C).

Sole treatment with PNGase is insufficient to completely deglycosylate sCLU but does promote changes within its secondary structure

To challenge the role of the core carbohydrates on sCLU chaperone activity, we decided to completely remove the $\mathrm{N}$-linked polysaccharides. Following the procedure published by Stewart et al. [16], we treated cleaved and uncleaved sCLU with PNGase at moderate and

Fig. 3. Approach for the full deglycosylation of cleaved sCLU. The deglycosylation was carried out by treatment of sCLU with different concentrations of PNGase F. A. Schematic drawing illustrating the cleavage site for PNGase F. B. Western blot analysis $(9 \%$ SDS-PAGE under non-reducing conditions) of cleaved sCLU treated with different concentrations of PNGase $F$. The detection of CLU was realized by using the anti-V5 antibody corresponding to the recombinant tag. The arrow indicates bands corresponding to remaining glycosylated sCLU. C. MALDI-TOF analysis of purified and PNGase F-treated sCLU. Qualitative analysis was performed with a sialic acid matrix.
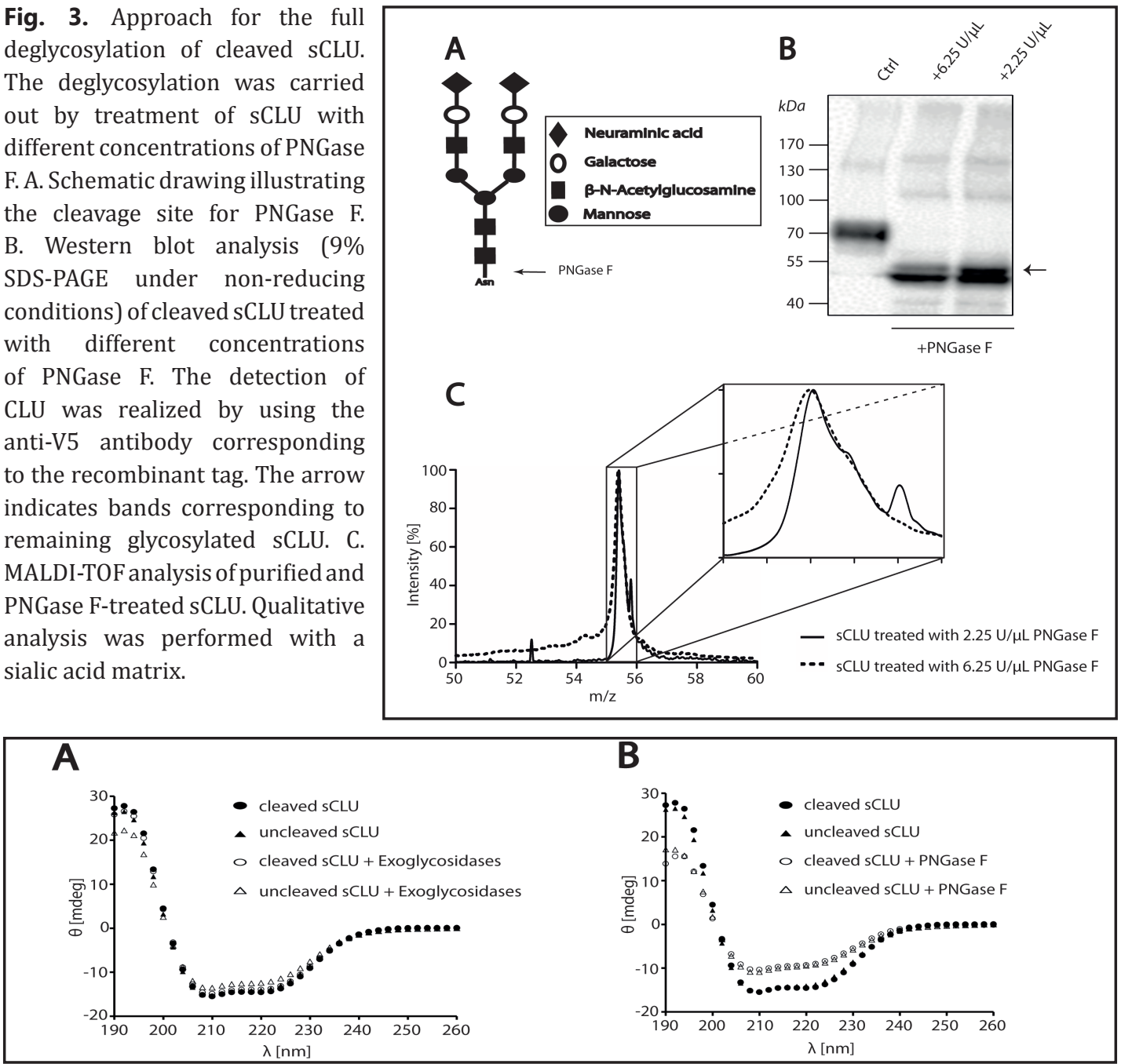

Fig. 4. CD-spectra of cleaved and uncleaved sCLU treated with or without glycosidases. Purified cleaved or uncleaved sCLU was either treated with or without $\alpha 2,3$-neuraminidase, $\beta 1,4$-galactosidase and $\beta$ - $N$-acetylglucosaminidase (A) or 6.25 U/ $\mu \mathrm{L}$ PNGase F (B) as described in Material and Methods. All CDspectra were obtained at wavelengths ranging from 190 to $260 \mathrm{~nm}$ in $1 \mathrm{~nm}$ intervals. The spectra shown illustrate the means of three consecutive scans and are representative for several individual experiments. Plotted is the ellipticity $\theta$ (mdeg) against the wavelength $\lambda(\mathrm{nm})$, whereas intervals of $2 \mathrm{~nm}$ were chosen for a better illustration. 
Tab. 1. Calculated percentage of secondary structure elements of cleaved and uncleaved sCLU in accordance with CD-spectra measurements ( \pm standard errors). The results were obtained from measurements carried out five to six times. Comparision between treated and untreated fractions were assessed by the mean \pm standard errors $\left({ }^{* * *} \mathrm{P}<0.001 ;{ }^{* *} \mathrm{P}<0.01 ;{ }^{*} \mathrm{P}<0.05\right)$

\begin{tabular}{|c|c|c|c|c|c|c|c|c|}
\hline & \multicolumn{2}{|c|}{$\alpha$-Helices } & \multicolumn{2}{|c|}{$\beta$-Sheets } & \multicolumn{2}{|c|}{ Turns } & \multicolumn{2}{|c|}{ Unordered } \\
\hline & Amount $[\%]$ & P-Value & Amount [\%] & P-Value & Amount $[\%]$ & P-Value & $\begin{array}{c}\text { Amount } \\
{[\%]}\end{array}$ & P-Value \\
\hline cleaved sCLU & $61.7 \pm 2.4$ & & $7.3 \pm 0.7$ & & $11.7 \pm 0.9$ & & $19.5 \pm 1.6$ & \\
\hline & & $* * *$ & & ** & & $* *$ & & $* *$ \\
\hline $\begin{array}{l}\text { cleaved sCLU } \\
+ \text { PNGase }\end{array}$ & $40.2 \pm 2.0$ & & $14.4 \pm 0.6$ & & $16.4 \pm 0.6$ & & $29.4 \pm 1.2$ & \\
\hline uncleaved sCLU & $60.8 \pm 2.3$ & & $9.3 \pm 0.9$ & & $12.0 \pm 0.7$ & & $17.8 \pm 1.7$ & \\
\hline & & $* * *$ & & $*$ & & * & & $* * *$ \\
\hline $\begin{array}{l}\text { uncleaved sCLU } \\
+ \text { PNGase }\end{array}$ & $40.4 \pm 2.4$ & & $14.6 \pm 1.3$ & & $16.4 \pm 0.7$ & & $29.0 \pm 1.0$ & \\
\hline
\end{tabular}

high concentrations. However, even at high concentrations a complete deglycosylation was not achieved. In Western blot analyses we still observed further sCLU- bands corresponding to protein species having residual glycosylation (Fig. 3B). Additionally, MALDI-TOF analyses revealed a prominent peak accompanying the main peak, demonstrating the presence of remaining carbohydrates (Fig. 3C). To evaluate the consequences of PNGase treatment on the structural organization of the proteins, the secondary structures were characterized using CD-spectroscopy (Fig. 4). As expected, the removal of distal carbohydrates upon treatment with exoglycosidases causes no significant alterations to the secondary structure. (Fig. 4A). However, in the case of PNGase-treated sCLU, the CD-spectra were altered in a more significant way (Fig. 4B). Analysis of the data predicted a decline of $\alpha$-helices with a concomitant increase in the amount of unordered regions after PNGase treatment (Tab. 1).

\section{Complete deglycosylation of sCLU can be achieved under reducing conditions}

Since full deglycosylation of sCLU was not achievable even at high PNGase concentrations (Fig. 3), we decided to use DTT to destabilize the protein conformation, hence allowing remaining carbohydrates to become more accessible to PNGase treatment. As shown by Western blot analysis, the $\alpha$ - and $ß$-chains display distinct sensitivities towards deglycosylation under reducing conditions (Fig. 5A). By using PNGase under non-reducing conditions, a remaining glycosylation could be observed on the $\beta$-chain of sCLU (Fig. 5A arrow upper panel), whereas after treatment in the presence of DTT, the $\beta$-chain was fully deglycosylated. In the latter case, however, not all carbohydrates on the $\alpha$-chain were removed (Fig. 5A arrow lower panel). A complete deglycosylation of both chains was only achieved by initially treating sCLU with PNGase for $6 \mathrm{~h}$ in the absence and subsequently $6 \mathrm{~h}$ in the presence of DTT (Fig. 5A). This procedure was also efficient when being applied to uncleaved sCLU. As expected, fully deglycosylated uncleaved sCLU displays an apparent molecular weight comparable to the intracellular $\mathrm{CLU}_{1-449}$ isoform [22] observed by overexpression of CLU cDNA variant 1 in HEK-293 cells (Fig. 5B). However, CLU $_{1-449}$ still carries the 22 amino acid ER-leader peptide hence its molecular weight differs slightly.

The core carbohydrates are critical for the chaperone activity of SCLU

After having generated fully deglycosylated sCLU, we set out to study its chaperone activity (Fig. 6A). To obtain a stable plateau phase between 90 and $150 \mathrm{~min}$ in the course of the measurement for evaluation, DTT was added to the assay as described in Material and Methods. The sequential PNGase/DTT treatment of cleaved sCLU (as depicted in Fig. 5) results in a reduction of the chaperone activity by approximately $80 \%$. This effect is even more pronounced regarding uncleaved sCLU where a reduction by $90 \%$ was measured upon full deglycosylation. Comparable results obtained from samples solely treated with PNGase, strengthen the suggestion that core carbohydrates are essential for sCLU chaperone activity. 


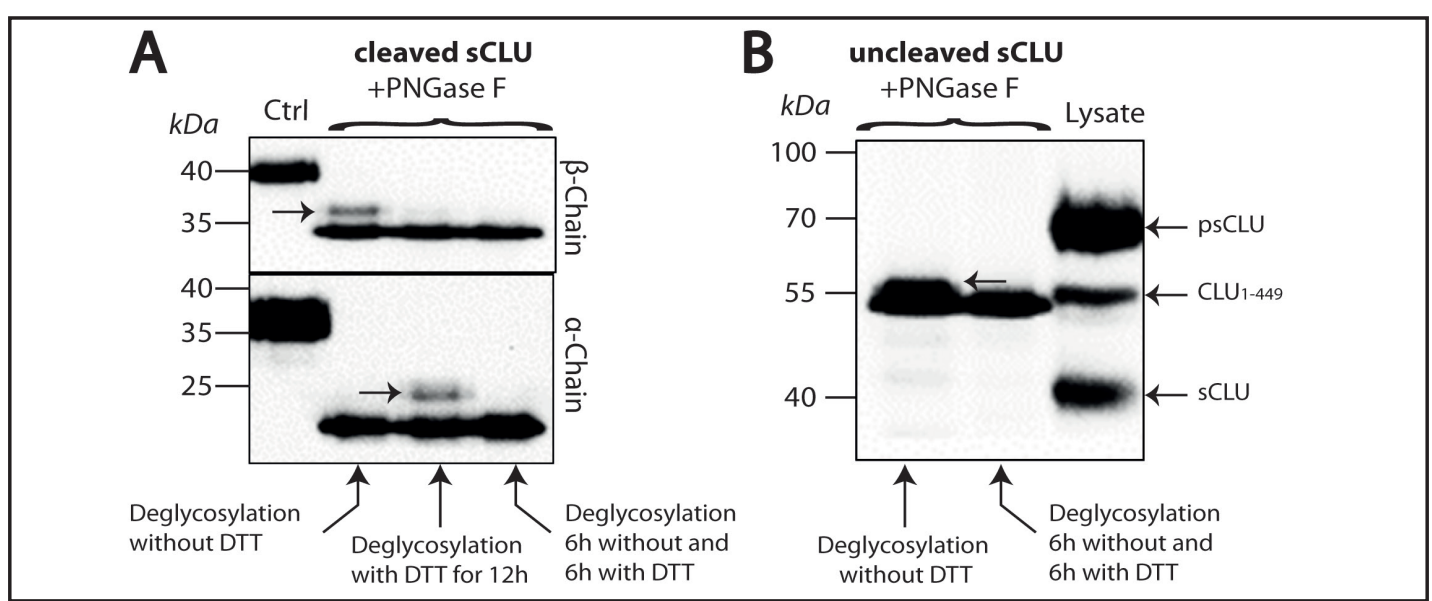

Fig. 5. Approach for full deglycosylation of cleaved and uncleaved sCLU. To obtain fully deglycosylated sCLU, three different strategies were tested: PNGase treatment without DTT for $12 \mathrm{~h}$, with $40 \mathrm{mM}$ DTT for $12 \mathrm{~h}$ or without DTT for $6 \mathrm{~h}$ and subsequent addition of $40 \mathrm{mM}$ DTT for another $6 \mathrm{~h}$. Western blot analysis of the reactions' products was performed under reducing conditions. Remaining glycosylated sCLU is indicated by arrows. A. Full deglycosylation of cleaved sCLU on $12.5 \%$ SDS-PAGEs. To detect the sCLU $\beta$-chain, the anti-V5 antibody and for the $\alpha$-chain the 41D antibody was used. B. Full deglycosylation of uncleaved sCLU was verified on a 9\% SDS-PAGE. To compare fully deglycosylated uncleaved sCLU with stress-induced unglycosylated non-secreted CLU forms, as described by Prochnow et al. [22], $50 \mu \mathrm{g}$ of lysates from HEK-293 cells transiently transfected with recombinant wild-type CLU constructs, were analyzed (see Fig. 1). The detection of CLU was realized by using the anti-V5 antibody corresponding to the recombinant tag. In the lysate lane, the most prominent band at $70 \mathrm{kDa}$ corresponds to the uncleaved sCLU precursor (psCLU) in the ER-Golgi-network. The apparent CLU band at $55 \mathrm{kDa}$ corresponds to the CLU form 1-449 which displays a mistranslocated CLU form still bearing the ER-Leader peptide.

The chaperone function of uncleaved CLU is sensitive to reducing conditions

As described in Fig. 5, a sequential DTT/PNGase treatment is necessary to obtain fully deglycosylated sCLU. We therefore used cleaved and uncleaved sCLU incubated in the presence of DTT as controls. Following this treatment, remarkably, a drop in sCLU chaperone activity down to approximately $15 \%$ was observed in case of the uncleaved form, whereas the activity of the cleaved form was merely lowered to $80 \%$ (Fig. 6A). Even by subjecting higher concentrations of both sCLU forms to different DTT concentrations, it becomes apparent that as little as $5 \mathrm{mM}$ DTT are already sufficient to impair the chaperone activity of uncleaved sCLU by one-third (Fig. 6B). Further, uncleaved sCLU incubated in the presence of $40 \mathrm{mM}$ DTT for $12 \mathrm{~h}$ shows a significant decrease by approximately 70\%. In the case of cleaved sCLU, DTT concentrations up to $40 \mathrm{mM}$ are still insufficient to decline the chaperone activity seriously, demonstrating the resistance of this sCLU form to reducing conditions (Fig. 6B). These data indicate that the chaperone activity of uncleaved sCLU is dependent on a nonreducing environment.

\section{Discussion}

Our investigations were prompted by reports that CLU accumulates in the cytosol under cellular stress $[49,52,53]$. As shown by Prochnow et al. [22], intracellular CLU forms might arise either from failure of the nascent polypeptide chain to be segregated into the ER, translation from a non-canonical start codon or alternative splicing events leading to a protein lacking the ER-leader peptide. Intracellular CLU generated by one of these mechanisms is unglycosylated and not proteolytically processed into the $\alpha$ - and the $ß$-subunit. However, intracellular CLU might also arise by retro-translocation from the ER-Golgi network into the 


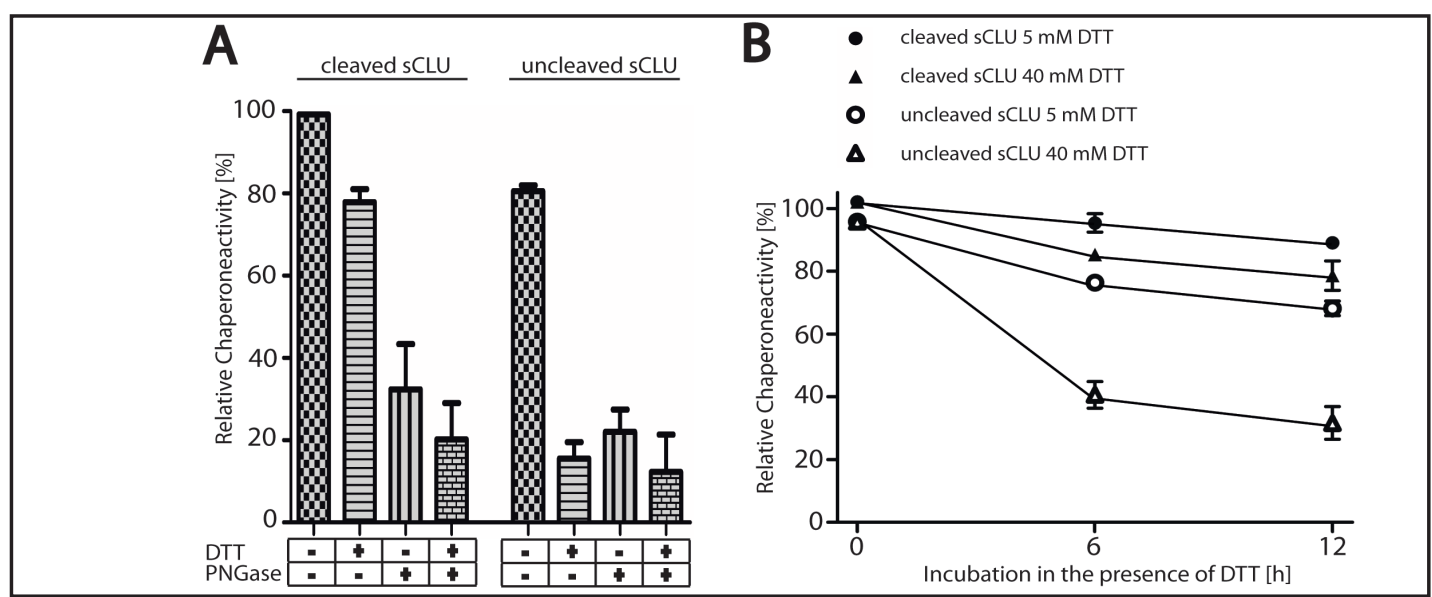

Fig. 6. Comparison of the chaperone activity of cleaved and uncleaved sCLU incubated with or without PNGase F and/or DTT. A. Cleaved and uncleaved sCLU were incubated either in the presence of $40 \mathrm{mM}$ DTT, with PNGase F or both with $40 \mathrm{mM}$ DTT and PNGase F to obtain fully deglycosylated sCLU (as shown in Fig. 5). The CLU fractions were subsequently mixed with Catalase for 150 min under reducing conditions ( 5 mM DTT). As a control, Catalase was mixed with BSA under the same conditions. The absorbance data obtained between 90 and $150 \mathrm{~min}$ (plateau phase) was taken as a measure for the relative chaperone activity. The BSA control is regarded as not being active as a molecular chaperone. Relative chaperone activity is expressed in relation to data obtained concerning untreated cleaved SCLU which is assumed to have a chaperone activity of $100 \%$. For the evaluation, the data of three independent experiments, each with the mean of three measurements were used. The error bars correspond to the mean \pm standard errors. B. Chaperone activity of cleaved and uncleaved sCLU incubated in the presence of 5 or 40 mM DTT for 0, 6 or $12 \mathrm{~h}$ at $37^{\circ} \mathrm{C}$ under gentle agitation. The assays and the analyses were carried out as described above. Data points are means of three measurements, error bars correspond to the standard deviation. The data shown are results of at least three independent experiments.

cytosol leading to hypoglycosylated intracellular CLU forms, which are also not been cleaved by furin-like proprotein convertases (PC) [7, 49]. This mechanism has also been described for the ER-Golgi chaperone Calreticulin [54]. It is currently not known whether the intracellular CLU forms exert chaperone activity such as secretory CLU (sCLU) $[1,50]$. Therefore, we set out to determine the need of the proteolytic processing and the glycosylation on the sCLU chaperone activity.

A common amino acid recognition motif for PCs is RxxR [55]. In the case of CLU it corresponds to the sequence RIVR, marking the border between $\alpha$ - and $\beta$-chain [7]. Hence, in a first experiment we generated HEK-293 cells stably transfected with CLU variant 1 cDNA, bearing a mutation in the PC cleavage site. Using this approach, we could demonstrate that substitution of the downstream arginine with glutamine (RIVR $\rightarrow$ RIVQ) completely abolished the proteolytic cleavage, enabling the generation of uncleaved sCLU. However, the maturation and secretion was not further affected by this modification, confirming earlier results indicating that the proteolytic processing of SCLU is not a prerequisite of secretion as shown by pharmacological inhibition of the proteolytic cleavage [8]. Subsequently, we purified uncleaved sCLU and wild-type (cleaved) sCLU proteins and used them for chaperone activity tests by applying either soluble cell extract as a complex or Catalase as a simple ligand. Irrespective of the ligand, we observed no significant difference between cleaved and uncleaved sCLU concerning their chaperone-active capacity. These results demonstrate that the proteolytic cleavage is not essential for the ability of sCLU to act as a molecular chaperone, a question recently pondered [50](Fig. 1).

To evaluate the role of the glycosylation on SCLU chaperone activity, we proceeded to prepare complete deglycosylated sCLU by the application of PNGase F. However, we realized that treatment of purified sCLU solely with PNGase did not result in a complete removal of the glycosylation, even at high enzyme concentrations. (Fig. 3). Although, the vast amount 
of sCLU was fully deglycosylated, minor amounts still possessed remaining carbohydrates, probably due to an inaccessibility of glycosylation sites for the endoglycosidase that may be caused by spontaneous protein aggregation [34,37]. To ensure full deglycosylation of sCLU, PNGase was used in the presence of DTT. PNGase treatment for $6 \mathrm{~h}$ followed by addition of DTT for another $6 \mathrm{~h}$ resulted in the full deglycosylation of sCLU (Fig. 5). Compared to glycosylated CLU, full deglycosylation resulted in a decrease in the chaperone activity by $70-90 \%$. No significant difference was observed between deglycosylated cleaved and uncleaved sCLU (Fig. 6A). These results led us to the conclusion that the non-glycosylated intracellular CLU-forms described by us and others [22, 56, 57] cannot exert chaperone activity due to the lack of polysaccharides. Hence, unglycosylated intracellular CLU may have a varying physiological function, probably by interacting with other intracellular proteins, such as Bax, Ku-70, SCLIP or Tau $[28,52,53,58]$. In addition, we could show that complete deglycosylation results in a significant alteration of the sCLU secondary structure. Especially the amount of $\alpha$-helices drops substantially (Fig. 4B, Tab. 1). In accordance with earlier suggestions, it can be concluded that changes in chaperone activity are accompanied by changes in the secondary structure [1]. In summary, our data are in contrast to reports which suggest that the deglycosylation has no impact on the chaperone activity and secondary structure of sCLU [16]. However, our findings do provide evidence that CLU forms retaining the core glycosylation show no significant alterations in the secondary structure or in their chaperone activity (Fig. 4A, Fig. 2). This observation bridges a gap to intracellular CLU forms, in particular to CLU forms emerging from retro-translocation of sCLU-precursors (i.e. psCLU) from ER-Golgi into the cytosol. Similar to the in vitro forms generated by us, these proteins are not proteolytically processed and exhibit an immature glycosylation pattern, i.e. they are hypoglycosylated. Hence, we conclude that these hypoglycosylated, intracellular CLU forms can also act as molecular chaperones. It has been discussed that intracellular CLU plays a role in maintaining the cellular homeostasis in pathological processes, such as ischemia, heat shock, ER-stress and other disorders [7, 24, 32, 49]. Our results imply that the chaperone activity could be one mechanism by which intracellular CLU might exert a cytoprotective function as long as at least the core carbohydrates are present.

Furthermore, as DTT was needed to generate completely deglycosylated sCLU, we also investigated the influence of DTT treatment on the chaperone activity. Intriguingly, the activity of uncleaved sCLU in a reducing environment was impaired by approximately $90 \%$ (Fig. 6A). Even under lower reducing conditions, a significant impairment was detectable. In contrast, this effect was not observed upon cleaved sCLU (Fig. 6B). The finding that uncleaved sCLU has a high sensitivity towards reducing conditions renders it unlikely to act as a chaperone in a reducing environment. On the basis of these data we postulate that the intracellular CLU forms are inactive in a reducing environment as present in the cytosol and only gain their activity under oxidative stress conditions or in subcellular areas having a varying reduction potential, such as mitochondria [24, 26, 59]. Hence, under oxidizing conditions, retro-translocated CLU may act as an intracellular molecular chaperone assisting the cell to cope with critical imbalances caused by inflammation and other pathological circumstances $[25,59]$. Furthermore, it is conceivable that retro-translocated CLU could act as a substitute for other intracellular chaperones which show an impaired activity under oxidative conditions. For instance, Hsp70 is considered to be inactivated upon oxidation, whereas the isoform Hsc70 is keeping up its activity [60]. In this context, it was shown that sCLU is capable of restoring the activity of unfolded proteins alongside Hsc70 in vitro [33]. Another chaperone, GRP78 (Bip), which is supposed to be an adapter for hypoglycosylated CLU is responsible for the retro-translocation of CLU from the ER-Golgi into the cytosol and to the mitochondria, respectively [49]. Intriguingly, the activity of Bip is tremendously altered upon oxidative stress, shifting its activity from an ATP-dependent foldase to an ATPindependent protein holdase [61]. This transition could promote an appropriate interaction with CLU. In summary, these three molecular chaperones (Bip, CLU and Hsc70) may be part of a proteostasis network $[24,25,43]$ to realize trafficking, binding and refolding of denatured intracellular proteins upon cellular stress conditions. 
Altogether, these considerations in line with our experimental findings provide strong evidence that only glycosylated CLU forms, regardless of their glycosylation pattern, are able to act as molecular chaperones supporting the cell in managing a plethora of stress situations. Retro-translocated CLU for its part is fine-tuned depending on the redox state of the cell and is silenced under non-pathological conditions. Moreover, the proteolytic cleavage is a key modification to maintain sCLU chaperone activity under reducing conditions, which might be encountered in tissues with ongoing cell damage or inflammation, such as atherosclerosis.

\section{Acknowledgement}

We thank Markus Rövekamp, Ilaria Montalbano and Georg Bündgen for their help with protein purification. Moreover, we thank Hildegard Pearson for carefully reading the manuscript and making helpful suggestions.

\section{References}

1 Humphreys DT, Carver JA, Easterbrook-Smith SB, Wilson MR: Clusterin has chaperone-like activity similar to that of small heat shock proteins. J Biol Chem 1999;274:6875-6881.

2 Wyatt A, Yerbury J, Poon S, Dabbs R, Wilson M: Chapter 6: The chaperone action of Clusterin and its putative role in quality control of extracellular protein folding. Adv Cancer Res 2009;104:89-114.

3 Jones SE, Jomary C: Clusterin. Int J Biochem Cell Biol 2002;34:427-431.

4 Falgarone G, Chiocchia G: Chapter 8: Clusterin: A multifacet protein at the crossroad of inflammation and autoimmunity. Adv Cancer Res 2009;104:139-170.

5 Choi-Miura NH, Takahashi Y, Nakano Y, Tobe T, Tomita M: Identification of the disulfide bonds in human plasma protein SP-40,40 (apolipoprotein-J). J Biochem 1992;112:557-561.

6 Sabatte J, Faigle W, Ceballos A, Morelle W, Rodriguez Rodrigues C, Remes Lenicov F, Thepaut M, Fieschi F, Malchiodi E, Fernandez M, Arenzana-Seisdedos F, Lortat-Jacob H, Michalski JC, Geffner J, Amigorena S: Semen clusterin is a novel DC-SIGN ligand. J Immunol 2011;187:5299-5309.

7 Nizard P, Tetley S, Le Drean Y, Watrin T, Le Goff P, Wilson MR, Michel D: Stress-induced retrotranslocation of clusterin/ApoJ into the cytosol. Traffic 2007;8:554-565.

$>8$ Urban J, Parczyk K, Leutz A, Kayne M, Kondor-Koch C: Constitutive apical secretion of an 80-kD sulfated glycoprotein complex in the polarized epithelial Madin-Darby canine kidney cell line. J Cell Biol 1987; 105:2735-2743.

-9 Lakins JN, Poon S, Easterbrook-Smith SB, Carver JA, Tenniswood MP, Wilson MR: Evidence that clusterin has discrete chaperone and ligand binding sites. Biochemistry 2002;41:282-291.

10 Blaschuk O, Burdzy K, Fritz IB: Purification and characterization of a cell-aggregating factor (clusterin), the major glycoprotein in ram rete testis fluid. J Biol Chem 1983;258:7714-7720.

11 Fritz IB, Murphy B: Clusterin Insights into a multifunctional protein. Trends Endocrinol Metab 1993;4:4145.

12 Matsubara E, Frangione B, Ghiso J: Characterization of apolipoprotein J-Alzheimer's A beta interaction. J Biol Chem 1995;270:7563-7567.

13 Wyatt AR, Yerbury JJ, Wilson MR: Structural characterization of clusterin-chaperone client protein complexes. J Biol Chem 2009;284:21920-21927.

- 14 Calero M, Tokuda T, Rostagno A, Kumar A, Zlokovic B, Frangione B, Ghiso J: Functional and structural properties of lipid-associated apolipoprotein J (clusterin). Biochem J 1999;344 Pt 2:375-383.

15 Hochgrebe T, Pankhurst GJ, Wilce J, Easterbrook-Smith SB: pH-dependent changes in the in vitro ligandbinding properties and structure of human clusterin. Biochemistry 2000;39:1411-1419.

-16 Stewart EM, Aquilina JA, Easterbrook-Smith SB, Murphy-Durland D, Jacobsen C, Moestrup S, Wilson MR: Effects of glycosylation on the structure and function of the extracellular chaperone clusterin. Biochemistry 2007;46:1412-1422.

17 Zlokovic BV, Martel CL, Matsubara E, McComb JG, Zheng G, McCluskey RT, Frangione B, Ghiso J: Glycoprotein 330/megalin: probable role in receptor-mediated transport of apolipoprotein J alone and in a complex with Alzheimer disease amyloid beta at the blood-brain and blood-cerebrospinal fluid barriers. Proc Natl Acad Sci U S A 1996;93:4229-4234. 
18 Dunker AK, Lawson JD, Brown CJ, Williams RM, Romero P, Oh JS, Oldfield CJ, Campen AM, Ratliff CM, Hipps KW, Ausio J, Nissen MS, Reeves R, Kang C, Kissinger CR, Bailey RW, Griswold MD, Chiu W, Garner EC, Obradovic Z: Intrinsically disordered protein. J Mol Graph Model 2001;19:26-59.

19 Fink AL: Natively unfolded proteins. Curr Opin Struct Biol 2005;15:35-41.

20 Loison F, Debure L, Nizard P, le Goff P, Michel D, le Drean Y: Up-regulation of the clusterin gene after proteotoxic stress: implication of HSF1-HSF2 heterocomplexes. Biochem J 2006;395:223-231.

21 Michel D, Chatelain G, North S, Brun G: Stress-induced transcription of the clusterin/apoJ gene. Biochem J 1997;328:45-50.

22 Prochnow H, Gollan R, Rohne P, Hassemer M, Koch-Brandt C, Baiersdorfer M: Non-secreted clusterin isoforms are translated in rare amounts from distinct human mRNA variants and do not affect Baxmediated apoptosis or the NF-kappaB signaling pathway. PLoS One 2013;8:e75303.

23 Richter K, Haslbeck M, Buchner J: The heat shock response: life on the verge of death. Mol Cell 2010;40:253-266.

24 Trougakos IP: The molecular chaperone apolipoprotein J/clusterin as a sensor of oxidative stress: implications in therapeutic approaches - a mini-review. Gerontology 2013;59:514-523.

25 Niforou K, Cheimonidou C, Trougakos IP: Molecular chaperones and proteostasis regulation during redox imbalance. Redox Biol 2014;2:323-332.

26 Kalyanaraman B: Teaching the basics of redox biology to medical and graduate students: Oxidants, antioxidants and disease mechanisms. Redox Biol 2013;1:244-257.

27 Bell RD, Sagare AP, Friedman AE, Bedi GS, Holtzman DM, Deane R, Zlokovic BV: Transport pathways for clearance of human Alzheimer's amyloid beta-peptide and apolipoproteins E and J in the mouse central nervous system. J Cereb Blood Flow Metab 2007;27:909-918.

28 Zhou Y, Hayashi I, Wong J, Tugusheva K, Renger JJ, Zerbinatti C: Intracellular clusterin interacts with brain isoforms of the bridging integrator 1 and with the microtubule-associated protein tau in Alzheimer's disease. PLoS One 2014;9:e103187.

29 Wehrli P, Charnay Y, Vallet P, Zhu G, Harmony J, Aronow B, Tschopp J, Bouras C, Viard-Leveugle I, French LE, Giannakopoulos P: Inhibition of post-ischemic brain injury by clusterin overexpression. Nat Med 2001;7:977-979.

-30 Han BH, DeMattos RB, Dugan LL, Kim-Han JS, Brendza RP, Fryer JD, Kierson M, Cirrito J, Quick K, Harmony JA, Aronow BJ, Holtzman DM: Clusterin contributes to caspase-3-independent brain injury following neonatal hypoxia-ischemia. Nat Med 2001;7:338-343.

-31 Sala A, Bettuzzi S, Pucci S, Chayka O, Dews M, Thomas-Tikhonenko A: Regulation of CLU gene expression by oncogenes and epigenetic factors implications for tumorigenesis. Adv Cancer Res 2009;105:115-132.

-32 Trougakos IP, Gonos ES: Chapter 9: Oxidative stress in malignant progression: The role of Clusterin, a sensitive cellular biosensor of free radicals. Adv Cancer Res 2009;104:171-210.

-33 Poon S, Easterbrook-Smith SB, Rybchyn MS, Carver JA, Wilson MR: Clusterin is an ATP-independent chaperone with very broad substrate specificity that stabilizes stressed proteins in a folding-competent state. Biochemistry 2000;39:15953-15960.

-34 Poon S, Treweek TM, Wilson MR, Easterbrook-Smith SB, Carver JA: Clusterin is an extracellular chaperone that specifically interacts with slowly aggregating proteins on their off-folding pathway. FEBS Lett 2002;513:259-266.

-35 Bailey RW, Dunker AK, Brown CJ, Garner EC, Griswold MD: Clusterin, a binding protein with a molten globule-like region. Biochemistry 2001;40:11828-11840.

-36 Law GL, Griswold MD: Activity and form of sulfated glycoprotein 2 (clusterin) from cultured Sertoli cells, testis, and epididymis of the rat. Biol Reprod 1994;50:669-679.

37 Poon S, Rybchyn MS, Easterbrook-Smith SB, Carver JA, Pankhurst GJ, Wilson MR: Mildly acidic pH activates the extracellular molecular chaperone clusterin. J Biol Chem 2002;277:39532-39540.

-38 Bartl MM, Luckenbach T, Bergner 0, Ullrich 0, Koch-Brandt C: Multiple receptors mediate apoJ-dependent clearance of cellular debris into nonprofessional phagocytes. Exp Cell Res 2001;271:130-141.

-39 Kounnas MZ, Loukinova EB, Stefansson S, Harmony JA, Brewer BH, Strickland DK, Argraves WS: Identification of glycoprotein 330 as an endocytic receptor for apolipoprotein J/clusterin. J Biol Chem 1995;270:13070-13075. 
Rohne et al.: Clusterin is Dependent on Glycosylation

40 Leeb C, Eresheim C, Nimpf J: Clusterin is a ligand for apolipoprotein E receptor 2 (ApoER2) and very low density lipoprotein receptor (VLDLR) and signals via the Reelin-signaling pathway. J Biol Chem 2014;289:4161-4172.

41 Yerbury JJ, Stewart EM, Wyatt AR, Wilson MR: Quality control of protein folding in extracellular space. EMBO Rep 2005;6:1131-1136.

42 Wyatt AR, Yerbury JJ, Berghofer P, Greguric I, Katsifis A, Dobson CM, Wilson MR: Clusterin facilitates in vivo clearance of extracellular misfolded proteins. Cell Mol Life Sci 2011;68:3919-3931.

43 Trougakos IP, Sesti F, Tsakiri E, Gorgoulis VG: Non-enzymatic post-translational protein modifications and proteostasis network deregulation in carcinogenesis. J Proteomics 2013;92:274-298.

44 Choi B, Kang SS, Kang SW, Min BH, Lee EJ, Song DH, Kim SM, Song Y, Yoon SY, Chang EJ: Secretory clusterin inhibits osteoclastogenesis by attenuating M-CSF-dependent osteoclast precursor cell proliferation. Biochem Biophys Res Commun 2014.

-45 Jun HO, Kim DH, Lee SW, Lee HS, Seo JH, Kim JH, Kim JH, Yu YS, Min BH, Kim KW: Clusterin protects H9c2 cardiomyocytes from oxidative stress-induced apoptosis via Akt/GSK-3beta signaling pathway. Exp Mol Med 2011;43:53-61.

-46 Shim YJ, Shin YJ, Jeong SY, Kang SW, Kim BM, Park IS, Min BH: Epidermal growth factor receptor is involved in clusterin-induced astrocyte proliferation. Neuroreport 2009;20:435-439.

47 Shin YJ, Kang SW, Jeong SY, Shim YJ, Kim YH, Kim BM, Kee SH, Park JJ, Park IS, Min BH: Clusterin enhances proliferation of primary astrocytes through extracellular signal-regulated kinase activation. Neuroreport 2006;17:1871-1875.

-48 Zhang B, Zhang K, Liu Z, Hao F, Wang M, Li X, Yin Z, Liang H: Secreted clusterin gene silencing enhances chemosensitivity of a549 cells to cisplatin through AKT and ERK1/2 pathways in vitro. Cell Physiol Biochem 2014;33:1162-1175.

49 Li N, Zoubeidi A, Beraldi E, Gleave ME: GRP78 regulates clusterin stability, retrotranslocation and mitochondrial localization under ER stress in prostate cancer. Oncogene 2013;32:1933-1942.

50 Dabbs RA, Wilson MR: Expression and purification of chaperone-active recombinant clusterin. PLoS One 2014;9:e86989.

51 Wong P, Taillefer D, Lakins J, Pineault J, Chader G, Tenniswood M: Molecular characterization of human TRPM-2/clusterin, a gene associated with sperm maturation, apoptosis and neurodegeneration. Eur J Biochem 1994;221:917-925.

52 Trougakos IP, Lourda M, Antonelou MH, Kletsas D, Gorgoulis VG, Papassideri IS, Zou Y, Margaritis LH, Boothman DA, Gonos ES: Intracellular clusterin inhibits mitochondrial apoptosis by suppressing p53-activating stress signals and stabilizing the cytosolic Ku70-Bax protein complex. Clin Cancer Res 2009;15:48-59.

53 Zhang H, Kim JK, Edwards CA, Xu Z, Taichman R, Wang CY: Clusterin inhibits apoptosis by interacting with activated Bax. Nat Cell Biol 2005; 7:909-915.

54 Afshar N, Black BE, Paschal BM: Retrotranslocation of the chaperone calreticulin from the endoplasmic reticulum lumen to the cytosol. Mol Cell Biol 2005;25:8844-8853.

55 Duckert P, Brunak S, Blom N: Prediction of proprotein convertase cleavage sites. Protein Eng Des Sel 2004;17:107-112.

56 Leskov KS, Klokov DY, Li J, Kinsella TJ, Boothman DA: Synthesis and functional analyses of nuclear clusterin, a cell death protein. J Biol Chem 2003;278:11590-11600.

57 Reddy KB, Jin G, Karode MC, Harmony JA, Howe PH: Transforming growth factor beta (TGF beta)-induced nuclear localization of apolipoprotein J/clusterin in epithelial cells. Biochemistry 1996;35:6157-6163.

-58 Kang SW, Shin YJ, Shim YJ, Jeong SY, Park IS, Min BH: Clusterin interacts with SCLIP (SCG10-like protein) and promotes neurite outgrowth of PC12 cells. Exp Cell Res 2005;309:305-315.

59 Mari M, Colell A, Morales A, von Montfort C, Garcia-Ruiz C, Fernandez-Checa JC: Redox control of liver function in health and disease. Antioxid Redox Signal 2010;12:1295-1331.

60 Miyata Y, Rauch JN, Jinwal UK, Thompson AD, Srinivasan S, Dickey CA, Gestwicki JE: Cysteine reactivity distinguishes redox sensing by the heat-inducible and constitutive forms of heat shock protein 70 . Chem Biol 2012;19:1391-1399.

61 Wang J, Pareja KA, Kaiser CA, Sevier CS: Redox signaling via the molecular chaperone BiP protects cells against endoplasmic reticulum-derived oxidative stress. Elife 2014;3:e03496. 